\title{
Index to Volume 129
}

\section{Compiled by William Halliday}

Activity,

Diurnal, 76

Nocturnal, 76

Alaska, 263

Alberta, 254

Kananaskis Country, 15

Rockies, 254

Western, 15

Alca torda, 152

Alcids, 152

Algae, 24

Allan, M., 277

Allan, M.R. The Use of Ketamine-Xylazine and KetamineMedetomidine With and Without Their Antagonists Yohimbine and Atipamezole Hydrochloride to Immobilize Raccoons (Procyon lotor) in Ontario, Canada, 84-89

Allison, E., 418

Alvar, 38, 45, 183

Ameiurus nebulosus, 189

Amphibian, 395 Larvae, 403

Analysis(es),

Genetic, 386

Multivariate, 121

Population Viability, 139

Anas,

clypeata, 323

discors, 323

platyrhynchos, 323

strepera, 323

Anderson, L., 277

Apeltes quadracus, 121

Aphrocallistes vastus, 399

Appetite, 374

Arachnida, 134

Arctic, 24, 134

High, 194

Areas, Moulting, 229

Arethusa bulbosa, 349

Aréthuse bulbeuse, 349

Assemblage, Fish, 121, 165

Atipamezole, 84

Barber-Meyer, S., 368

Bay,

Hudson, 229

Ungava, 229

Bear,

American Black, 8

Grizzly, 8

Bee, Mason, 38

Behaviour,

Elk, 282

Feeding, 374

Hunting, 368

Nesting, 245

Biodiversity, 15

Biogeography, 134

Island, 139

Biomanipulation, 165
$\operatorname{Bird}(\mathrm{s})$,

Grassland, 323

Sea, 152

Blowdown, 285

Boar, Wild, 76

Bobolink, 323

Bonasa umbellus, 245

Bond, A.L., J.L. Lavers. Flesh-footed Shearwaters (Puffinus carneipes) in the Northeastern Pacific Ocean: Summary and Synthesis of Records from Canada and Alaska, 263-267

Booby, Brown, 53

Boswell, R. New Light on the Origins of the Ottawa FieldNaturalists' Club, 207-213

Branta,

canadensis, 229, 290

hutchinsii, 194

Breeding, 194

Bremner, A.M., D.A. Methven, K.R. Munkittrick, K.A. Frego. Spatial and Temporal Variation in Fish Assemblages in Three Small Unpolluted Estuarine Rivers and Associated Lagoons in Kouchibouguac National Park, Southern Gulf of St. Lawrence, Canada, 121-133

British Columbia, 263

Haida Gwaii, 285

Howe Sound, 399

Southwestern, 159

Brodo, I., 415

Brood-rearing, 229

Brook, R.K., 76, 403

Brook, R.K. Observations of the Use of Buildings by Freeranging Elk, Cervus canadensis, in Prince Albert National Park, Saskatchewan, 282-284

Brooks, R.J., 189

Brown, A., 285

Brown, D.P., 139

Brunton, D.F., J. McNeill. Status, Distribution, and Nomenclature of Northern Quillwort, Isoetes septentrionalis (Isoetaceae), in Canada, 174-180

Brunton, D.F. Great Plains Ladies'-tresses (Spiranthes magnicamporum) in the Lower Great Lakes Region and a New Record for New York State, 183-188

Buddle, C.M. Life History and Distribution of the Arctic Pseudoscorpion, Wyochernes asiaticus (Chernetidae), 134138

Buildings, 282

Bullfrog,

American, 395

Blue, 395

Bullhead, Brown, 189

Calidris,

alpina, 159

melanotos, 194

Callaghan, C., T. Rytwinski. Editor's Report for Volume 128

Camera, (2014), 216-218

Infrared, 245

Trail, 76

Campbell, D., 277

Canadian Museum of Nature, 24

Note: Page references are to the first page of the item (e.g., article, note), and as such are not always to the exact word location. 
Canis,

Latrans, 386

lupus, 290, 368, 386

lycaon, 386

Carex,

brunnescens subsp. sphaerostachya, 349 pauciflora, 349

Carex,

à épis globulaires, 349

pauciflore, 349

Carruthers, M., 115

Cascade, Trophic, 165

Catling, P.M., B. Kostiuk, D. Thompson. Horse Ranching Increases Biodiversity in a Foothills Parkland Prairie in Northern Kananaskis Country, Western Alberta, 1523

Catling, P.M. Osmia species (Megachilidae) Pollinate Cypripedium parviflorum (Orchidaceae) and Packera paupercula (Asteraceae): A Localized Case of Batesian Mimicry?, 38-44

Catrysse, J., E. Slavik, J. Choquette, A.E. Leifso, C.M. Davy. Mass Mortality of Northern Map Turtles (Graptemys geographica), 80-83

Cattle, 331

Cermack, R., 419

Cervus canadensis, 282

Chelydra serpentina, 189, 403

Choquette, J., 80

Christmas Bird Count, 159

Chrosomus eos, 237

Chrysemys picta marginata, 403

Cipriani, J., 415

Citizen Science, 254, 263, 342

Clayden, S.R., 219

Cliffbrake, Gastony's, 45

Climate Change, 139, 254

Cobb, E., 115

Collection, 24

Community, Structure, 331

Connectivity, 139

Conservation, 386

Conservation Award - Member: Anouk Hoedeman, 417

Conservation Award - Non-Member: Paul and Cathy Keddy, 417

Constancy, Incubation, 245

Cook, F.R. Timothy Christopher ("CHRIS") Brayshaw 19192014,114

Coregoninae, 70

Cotter, R., 152

Cotter, R.C., Distribution and Use of Brood-rearing and Moulting Sites of the Atlantic Population of Canada Geese (Branta canadensis) in Nunavik, Quebec, 229236

Cougar, 277

Cowbird, Brown-headed, 60

Coyote, 386

Cygnets, 374

Cygnus,

buccinator, 374

columbianus, 194

Cypripedium parviflorum var. pubescens, 38

DNA, 277

Extraction, 53

Identification, 53

Mitochondrial, 53

Dace, Northern Redbelly, 237

Damage, Tissue, 399

Darter, Iowa, 237

Davy, C.M., 80
Deer, White-tailed, 1, 368

Defense, Prey, 189

Dekker, D., M.C. Drever. Kleptoparasitism by Bald Eagles (Haliaeetus leucocephalus) as a Factor Reducing Peregrine Falcon (Falco peregrinus) Predation on Dunlin (Calidris alpina) Wintering in British Columbia, 159164

Departure, Chick, 285

Devices, Automated Recording, 115

Diet, 189, 403

Dispersal, 181, 229 Seed, 403

Distribution, 53, 70, 134, 174, 268, 342

Disturbance, 285 Intermediate, 331

Dobony, C.A., 245

Dolichonyx oryzivorus, 323

Draba glabella, 24

Drawdown, 374

Drever, M.C., 159

Driscoll, K.E., 219

Dumping, Egg, 60

Dunlin, 159

Dynamics, Patch, 15

Eagle, Bald, 159

Ecology, Incubation, 245

Edwards, J.W., 245

Effect, Top-Down, 165

El Niño, 399

Elk, 282

Endangered, 277

Epilobium brachycarpum, 268

Equus ferus caballus, 15

Erethizon dorsatum, 273

Esocidae, 165

Esox lucius, 165

Estuary, 121

Fraser River, 159

Etheostoma exile, 237

Evenness, 15

Experimental Lakes Area, 165

Extension, Range, 70, 183, 237, 349

Falco peregrinus, 159

Falcon, Peregrine, 159

Fawn, 368

Feces, 53

Fecundity, 134

Fern, 45

Fescue,

Grassland, 15

Mountain Rough, 15

Festuca, campestris, 15

edlundiae, 24

Fidelity, Site, 379

Fish(es), 121

Floristique, 349

Foothills, Prairie, 15

Foraging, 331, 374, 403

Fratercula arctica, 152

Frego, K.A., 121

Friesen, C., C. Murray. Gastony's Cliffbrake (Pellaea gastonyi) in Manitoba: New Records and Assessment of Fundulus, Conservation Status, 45-52

Fungi, 24

diaphanus, 121

heteroclitus, 121

Gadwall, 323 
Garon-Labrecque, M.-È., É. Léveillé-Bourret, K. Higgins, O. Sonnentag. Additions to the Boreal Flora of the Northwest Territories with a Preliminary Vascular Flora of Scotty Creek, 349-367

Gasterosteus, aculeatus, 121 wheatlandi, 121

Gaston, A.J., J. Pattison, A. Brown. Impact of a Major Forest Blowdown Event on Breeding of Ancient Murrelets, Synthliboramphus antiquus, at a Colony in Haida Gwaii, British Columbia, 285-289

Geographic Information System, 331, 342

George McGee Service Award: Elizabeth Morton, 416

Gilhen, J., R.W. Russell. Three Records of Rare Blue American Bullfrogs, Lithobates catesbeianus, in Nova Sco-

Gillnet, 70 tia, Canada, 395-398

Giroux, M.-A., 194

Gitzen, R.A., 139

Goertz, D.E., R.D. Phoenix. Range Extensions for the Northern Redbelly Dace (Chrosomus eos), Fathead Minnow (Pimephales promelas), and Iowa Darter (Etheostoma exile) in Ontario, Canada, 237-244

Goltz, B.N., 386

Goose,

Cackling, 194

Canada, 229, 290

Gradient(s),

Spatial, 121

Temporal, 121

Grant, T.A., T.L. Shaffer. Do Ducks and Songbirds Initiate More Nests When the Probability of Survival is Greater?, 323-330

Graptemys geographica, 80

Grass, 374

Grazing, 15, 331

Ground Squirrel, Richardson's, 331

Grouse, Ruffed, 245

Gulf of St. Lawrence, 121, 152 North Shore, 152

Gulo gulo, 273

Haber, E. A Tribute to John Montague Gillett, 1918-2014

Habitat, 134

Habitat Loss, 45

Habituation, 282

Haliaeetus leucocephalus, 159

Hamer, D., I. Pengelly. Whitebark Pine (Pinus albicaulis) Seeds as Food for Bears (Ursus spp.) in Banff National Park, Alberta, 8-14

Handling, Prey, 189

Hanrahan, C., 415

Hare, J.F., 331

Healing, 399

Herman, T.B., 379

Heterogeneity, 15

Higgins, K., 349

Hoedeman, A., 417

Holmes, S.B., K. Tuininga, K.A. Mcllwrick, M. Carruthers, E. Cobb. Using an Integraed Recording and Sound Analysis System to Search for Kirtland's Warbler (Setophaga kirtlandii) in Ontario, 115-120

Home Range, 1, 379

Honorary Member: Ross Layberry, 415

Horse(s), 15 Feral, 15

Hunting, Cooperative, 290

Hydroprogne caspia, 152

Identification, 268
Imlay, T.L., J. Saroli, T.B. Herman, S.W. Mockford. Movements of the Eastern Ribbonsnake (Thamnophis sauritus) in Nova Scotia, 379-385

Immobilization, Chemical, 84

Incompatibility, Host, 60

Incubation, 245

Initiation, Nest, 323

Inlet, Cunningham, 24

Invasion, Top Predator, 165

Island(s),

Hutt, 399

Igloolik, 194

Queen Charlotte, 285

Somerset, 24

Isle Royale, 139

Isoetes,

canadensis var. robbinsii, 174

riparia, 174

septentrionalis, 174

$\mathrm{x}$ didgei, 174

Isotopes, Stable, 386

Jacklin, M., 53

Jung, T.S., 273

Keddy, C., 417

Keddy, P., 417

Keevil, M.G., 189, 403

Kell, S., 189

Kelso, S., 53

Kennedy, J., 386

Kerr, K.C.R., 53

Ketamine, 84

Khan, T., 277

Killifish, Banded, 121

Kittiwake, Black-legged, 152

Kleptoparasitism, 159

Kostiuk B., 15

Kukka, P.M., T.S. Jung. The Cost of a Prickly Diet: Incidents of Porcupine Erethizon dorsatum) Quills Embedded in Wolverine (Gulo gulo), 273-277

La Niña, 399

Ladies'-tresses, Great Plains, 183

Lady's-slipper, Large Yellow, 38

Lagoon, 121

Lake(s),

Bluefish, 70

Erie, 80

Grafton, 379

Great, 53

Great Slave, 70

Molega, 379

Superior, 139

Yellowknife, 70

Larids, 152

Lavers, J.L., 263

Layberry, R., 415

Laying, Emergency Egg, 60

Lecomte, N., M.-A. Giroux. New Avian Breeding Records for Igloolik Island, Nunavut, 194-196

Leifso, A.E., 80

Léveillé-Bourret, É., 349

Lichens, 24, 219

Licht, D.S., R.A. Moen, D.P. Brown, M.C. Romanski, R.A. Gitzen. The Canada Lynx (Lynx canadensis) of Isle Royale: Over-harvest, Climate Change, and the ExtirLimestone, 45 pation of an Island Population, 139-151

Limit, Range, 263

Lithobates catesbeianus, 395 
Litzgus, J.D., 189, 403

Lowlands, Hudson Bay, 237

Lumsden, H.G., V.G. Thomas, B.W. Robinson. Response of Wild Trumpeter Swan (Cygnus buccinator) Broods to Wetland Drawdown and Changes in Food Abundance, 374-378

Lynx, Canada, 139

Lynx canadensis, 139

MacDonald, C., 277

MacDonald, I.D., 268

MacKenzie, A., 415

Maine, 181

Mallard, 323

Management, 15 Fishery, 165

Manipulation, Food Web, 165

Manitoba, Interlake Region, 45 Westbourne, 331

Mark-Recapture, 165

Marliave, J. Cloud Sponge, Aphrocallistes vastus (Porifera: Hexactinellida), Fragment Healing and Reattachment, 399-402

Mary Stuart Education Award: Tom Spears, 418

McAlpine, D.F., D.X. Soto, L.Y. Rutledge, T.J. Wheeldon, B.N. White, J.P. Goltz, J. Kennedy. Recent Occurrences of Wild-origin Wolves (Canis spp.) in Canada South of the St. Lawrence River Revealed by Stable Isotope and Genetic Analysis, 386-394

McCarthy, J.W., K.E. Driscoll, S.R. Clayden. Lichens in Four Newfoundland Provincial Parks: New Provincial Records, 219-228

McIlwrick, K.A., 115

McKinney, S.T., 181

McLachlan Hamilton, K., 415

McNeill, J., 174

Mech, L.D., A. Morris, S. Barber-Meyer. White-tailed Deer (Odocoileus virginianus) Fawn Risk from Gray Wolf (Canis lupus) Predation During Summer, 368-373

Medetomidine, 84

Member of the Year: Natalie Sopinka, 416

Menidia menidia, 121

Metapopulation, 139

Methven, D.A., 121

Midden, 8

Migration, Vertical, 254

Mills, K.H., 165

Mills, P.B., 403

Mimicry, Floral, 38

Minnesota, 139 Ely, 368

Northeastern, 1

Minnow, Fathead, 237

Minutes of the $136^{\text {th }}$ Annual Business Meeting of the Ottawa Field-Naturalists' Club January 13, 2015, 310-322

Mirris, A., 368

Mockford, S.W., 379

Model, Climate, 254

Moen, R.A., 139

Moldowan, P.D., M.G. Keevil, S. Kell, R.J. Brooks, J.D. Litzgus. Anti-predator Defenses of Brown Bullheads ( $\mathrm{Am}$ eiurus nebulosus) and Interactions with Snapping Turtles (Chelydra serpentina), 189-193

Moldowan, P.D., M.G. Keevil, P.B. Mills, R.J. Brooks, J.D. Litzgus. Diet and Feeding Behaviour of Snapping Turtles (Chelydra serpentina) and Midland Painted Turtles (Chrysemys picta marginata) in Algonquin Provincial Park, Ontario, 403-408
Molothrus ater, 60

Mortality, Mass, 80

Morton, E., 416

Mosaic, Shifting, 15

Mosses, 24

Moulting, 229

Mountains, Appalachian, 245

Mouse, Deer, 181

Movement(s), 1, 181, 379

mtDNA, 53

Mummichog, 121

Munkittrick, K.R., 121

Murray, C., 45

Murrelet, Ancient, 285

Murres, Common, 152

National Forest, Superior, 1, 368

National Historic Site, Kejimkujik, 379

National Park,

Banff, 8

Kejimkujik, 379

Kouchibouguac, 121

Prince Albert, 282

National Wildlife Refuge, Salyer, 323

Natural History, 134

Necropsy, 273, 386

Nelson, M.E. Experimental Evidence of Spatial Memory and Home Range Affinity in White-tailed Deer (Odocoileus virginianus), 1-7

Nest,

Initiation, 323

Survival, 323

New Brunswick, 386

New York, Jefferson County, 183

Newediuk, L.J., I. Waters, J.F. Hare. Aspen Parkland Pasture Altered by Richardson's Ground Squirrel (Urocitellus richardsonii Sabine) Activity: The Good, the Bad, and the Not So Ugly?, 331-341

Newfoundland, 219

Nichols, T.C. Cooperative Hunting of Canada Geese (Branta canadensis) by Gray Wolves (Canis lupus) in Northern Quebec, 290-292

Nicholson, M.E., M.D. Rennie, K.H. Mills. Apparent Extirpation of Prey Fish Communities Following the Introduction of Northern Pike (Esox lucius), 165-173

Nomenclature, 174

North Dakota, 323

Northwest Territories, 70, 134, 349

Fort Simpson, 349

Scotty Creek, 349

Nova Scotia, 379, 395

Nunavut, 24 Igloolik, 194

Nutrients, 374

Obituary, 114

Observation, Behavioural, 159

Oceanodroma leucorhoa, 152

Ochotona princeps, 254

Odocoileus virginianus, 1, 368

Odonate, 403

Oldham, M.J., I.D. MacDonald. Tall Annual Willowherb (Epilobium brachycarpum C. Presl) in Ontario, Canada, 268-272

Onagraceae, 268

Ontario, 237, 268, 277, 342

Algoma District, 115

Aurora, 374 
Eastern, 38, 183

Great Lakes, 53

Hudson Bay Lowlands, 237

Northern, 115, 237

Ottawa, 183

Northwestern, 165

Southwestern, 80

Osmia, 38

Ottawa Field-Naturalists' Club Awards for 2014, presented April 2015, 415-419

Ottawa Field-Naturalists' Club Minutes of the $136^{\text {th }}$ Annual Business Meeting, 310-322

Ouellet, C., 277

Over-harvest, 139

Packera paupercula, 38

Panayi, D., 70

Parasitism, Brood, 60 Multiple, 60

Parkland, Aspen, 331

Passerculus sandwichensis, 323

Passerine, 323

Pattison, J., 285

Pawlicki, J., 53

Pellaea gastonyi, 45

Pengelly, I., 8

Pepper, R., 277

Peromyscus maniculatus, 181

Phoenix, R.D., 237

Pigmentation, Dermal, 395

Pika, American, 254

Pike, Northern, 165

Pimephales promelas, 237

Pine, Whitebark, 8

Pinus albicaulis, 8

Plants,

Rare, 349

Vascular, 15, 24, 268

Platanthera dilatata var. dilatata, 349

Platanthère dilatée, 349

Pollination, 38

Pond, Ephemeral, 403

Population(s), 152

Atlantic, 229

Meta-, 139

Porcupine, North American, 273

Predation, 159, 245, 290, 368

President's Prize: Elisabeth Allison, 418

President's Prize: Robert Cermack, 419

Puma, 277

Puma concolor, 277

Pungitius pungitius, 121

Procyon lotor, 84

Prosopium coulterii, 70

Provincial Park, 219

Algonquin, 189, 403

Bow Valley, 15

Fitzgerald's Pond, 219

J.T. Cheeseman, 219

Jipujijkuei Kuespem, 219

Sandbanks, 219

Pseudoscorpiones, 134

Puffin, Atlantic, 152

Puffinus carneipes, 263

Quadrats, 15

Québec, 386

Northern, 290

Quills, 273
Quillwort,

Northern, 174

Riverbank, 174

Raccoon, 84

Ragwort, Balsam, 38

Rail, J.-F., R. Cotter, Seventeenth Census of Seabird Populations in the Sanctuaries of the North Shore of the Gulf of St. Lawrence, 2010, 152-158

Range, 263 Breeding, 115

Rangeland, 15, 331

Razorbills, 152

Recapture, 379

Record, New, 53, 219

Relict, Post-Glacial, 183

Rennie, M.D., 165

Review, 60 Literature, 139

Ribbonsnake, Eastern, 379

Richness, 15

Rissa tridactyla, 152

River,

Black, 121

Fraser River, 159

Kouchibouguac, 121

Kouchibouguacis, 121

St. Lawrence, 386

Robinson, B.W., 374

Romanski, D.P., 139

Rosatte, E., L. Anderson, D. Campbell, C. Ouellet, B. White, T. Khan, P. Van Schyndel, R. Pepper, C. MacDonald, W. Wegman, M. Allan. Further Evidence of Cougars (Puma concolor) in Ontario, Canada, 277-281

Russell, R.W., 395

Rutledge, L.Y., 386

Rytwinski, T., 216

Sample, Fecal, 53

Sanctuaries, Bird, 152

Sandpiper, Pectoral, 194

Saroli, J., 379

Saskatchewan, Central, 76, 282

Sea, Champlain, 174

Seabirds, 152, 263

Sealy, S.G. Egg Laying in Inappropriate Nests by the Brownheaded Cowbird (Molothrus ater): Acts of Parasitism or Emergency Egg Dumping?, 60-69

Season, Non-breeding, 263

Seburn, D.C. Distribution of the Exotic Pond Slider (Trache-

Seeds, 8 mys scripta) in Ontario, 342-348

Seine, Beach, 121

Selection, Host, 60

Setophaga kirtlandii, 115

Shaffer, T.L., 323

Shank, C.C. Are American Pikas (Ochotona princeps) in the Canadian Rockies Vulnerable to Climate Change?, 254-262

Shearwater, Flesh-footed, 263

Shoveler, Northern, 323

Silverside, Atlantic, 121

Skevington, J.H., J. Pawlicki, S. Kelso, K.C.R. Kerr, M. Jacklin. Subspecific Identification of the Great Lakes' First Brown Booby (Sula leucogaster) Using DNA, 5359

Slavik, E., 80

Slider, Pond, 342

Smith, B.W., A.N. Tri, C.A. Dobony, J.W. Edwards, P.B. Wood. Behaviour and Nesting Ecology of Appalachian Ruffed Grouse (Bonasa umbellus), 245-253 
Snails, 374

Software, Sound Analysis, 115

Sokoloff, P.C. The Flora of Cunningham Inlet, Somerset Island, Nunavut: History, Analysis, and New Collections of Vascular Plants, Mosses, Lichens, and Algae, 24-37

Sonnentag, O., 349

Sopinka, N., 416

Soto, D.X., 386

Sparrow,

Clay-colored, 323

Savannah, 323

Spatial Affinity, 1

Spatial Memory, 1

Spears, T., 418

Species,

Invasive, $76,165,342$

Rare, 45

Spine, Pectoral, 189

Spiranthes magnicamporum, 183

Spizella pallida, 323

Sponge, Cloud, 399

Squirrel, Red, 8

Status, 268

Stickleback,

Blackspotted, 121

Fourspine, 121

Ninespine, 121

Threespine, 121

Stolle, K., F.M. van Beest, E. Vander Wal, R.K. Brook. Diurnal and Nocturnal Activity Patterns of Invasive Wild Boar (Sus scrofa) in Saskatchewan, Canada, 76-79

Storm-Petrel, Leach's, 152

Structure, Community, 331

Success,

Breeding, 285

Hatch, 245

Sula leucogaster, 53

Survey, 45, 323

Aerial, 8, 229

Counts, 159, 331

Fyke Net, 165

Gillnet, 70, 165

Helicopter, 229

Live-Trap, 181

Minnowtrap, 165

Quadrat, 331, 349

Snorkel, 165

Transect, 8, 15, 374

Survival,

Visual, 331, 349, 379

Nest, 323

Time-specific, 323

Sus scrofa, 76

Swan,

Trumpeter, 374

Tundra, 194

Swine, Feral, 76

Synthliboramphus antiquus, 285

Tamiasciurus hudsonicus, 8

Teal, Blue-winged, 323

Telemetry,
GPS, 1,8

Radio, 245, 368

Tern, Caspian, 152

Thamnophis sauritus, 379

Thomas, V.G., 374

Thompson, D., 15

Time, Laying, 60

Trachemys scripta, 342

Trade, Pet, 342

Translocation, Experimental, 1

Trap,

Camera, 76, 245

Lilly-pad, 245

Live-, 84, 145

Minnow, 237

Tri, A.N., 245

Tribute, 90

Tuininga, K., 115

Turtle, 342

Midland Painted, 403

Northern Map, 80

Snapping, 189, 403

Uria aalge, 152

Urocitellus richardsonii, 331

Ursus,

americanus, 8

arctos, 8

Vagrant, 53

Van Beest, F.M., 76

Van Schyndel, P., 277

Vander Wal, E., 76

Vecsei, P., D. Panayi. Range Extension for Pygmy Whitefish (Prosopium coulterii) in the Northwest Territories, Canada, 70-75

Vulnerability, 254

Warbler, Kirtland's, 115

Waterfowl, 323

Waters, I., 331

Wegman, W., 277

West Virginia, 245

Wheeldon, T.J., 386

White, B., 277

White, T.J., 386

Whitefish, Pygmy, 70

Willowherb, Tall Annual, 268

Wolf,

Eastern, 386

Gray, 290, 368, 386

Wolverine, 273

Wood, C.M., S.T. McKinney. Record Long-Distance Movement of a Deer Mouse, Peromyscus maniculatus, in a New England Montane Boreal Forest, 181-182

Wood, P.B., 245

Wyochernes asiaticus, 134

Xylazine, 84

Yohimbine, 84

Yukon Territory, 134

Zurbrigg, E., I. Brodo, J. Cipriani, C. Hanrahan, A. MacKenzie, K. McLachlan Hamilton. The Ottawa Field-Naturalists' Club Awards for 2014, presented April 2015, 415-419 


\section{Index to Book Reviews}

\section{Botany}

Brunton, D.F. "Flora of Virginia" by Alan S. Weakley, J. Christopher Ludwig, and John Townsend, 2012, 105106

Haber, E. "À la découverte du Nord: deux siècles et demi d'exploration de la flore nordique du Québec et du Labrador" by Jacques Cayouette, 2014, 200-201

John, R. "A Field Guide to Seaweeds of the Pacific Northwest" by Bridgette Clarkson, 2015, 409-410

McBride, B. "Trees of Western North America" by Richard Spellenberg et al., 2014, 201-202

\section{Other}

Armstrong, T. "Saving Global Fisheries. Reducing Fishing Capacity to Promote Sustainability" by J. Samuel Barkin and Elizabeth R. DeSombre, 2013, 303-304

Bocking, S. "Reclaiming the Don: An Environmental History of Toronto's Don River Valley" by Jennifer L. Bonnell, 2014, 302-303

Brooks, R. "Algonquin Park. A Portrait: The Landscape, Wildlife and Ecology of an Iconic Canadian Treasure" by Jan Rinik and Martin Rinik, 2014, 202-203

Houston, C.S. "Ian McTaggart-Cowan: The Legacy of a Pioneering Biologist, Educator and Conservationist" by Ronald D. Jakimchuk, R. Wayne Campbell, and Dennis A. Demarchi, 2014, 107-109

Houston, C.S. "The Curious Mister Catsby" by E. Charlson Nelson, David J. Elliott, et al., 2015, 300-301

Houston, C.S. "The New Labrador Papers of Captain George Cartwright" by Marianne P. Stopp, 2008, 411

John, R. New Titles, 111, 206, 305-306, 412

John, R. "Behind the Binoculars: Interviews with Acclaimed Birdwatchers" by Mark Avery and Keith Betton, 2015, 299-300

O’Neill, J. “Climber's Paradise: Making Canada's Mountain Parks, 1906-1974” by Pearlann Reichwein, 2014, 301302

Seburn, D. "Handbook of Road Ecology" by Rodney van der Ree, Daniel J. Smith, and Clara Grilo, 2015, 410-411

Shaver, J. "Rare - The High-Stakes Race to Satisfy Our Need for the Scarcest Metals on Earth" by Keith Veronese, 2014, 203-204

Smith, C.M. "Conrad Kain: Letters from a Wandering Mountain Guide, 1906-1933” by Zac Robinson, 2014, 109110

Stanton-Kennedy, T. "Transient Landscapes: Insights into a Changing Planet" by Ellen E. Wohl, 2015, 304-305

Tegler, B. "The Carbon Bubble" by Jeff Rubin, 2015, 410

Way, J. "My Watery Self: Memoirs of a Marine Scientist" by Stephen Spotte, 2015, 204-205

\section{Zoology}

Brooks, R. “The Passenger Pigeon” by Errol Fuller, 2014, 199200

Burke, T. "The R.O.M. Field Guide to Butterflies of Ontario" by Peter W. Hall et al., 2014, 197-198

Cook, F.R. "Amphibian Biology, Volume 11, Part 4: Status of Conservation and Decline of Amphibians: Eastern Hemisphere: Southern Europe \& Turkey" by Harold Heatwole and John W. Wilkinson, 2014, 97

Foster, R.F. "Running Silver - Restoring Atlantic Rivers and Their Great Fish Migrations" by John Waldman, 2013, 198-199

Gibson, J.F. "Arthropods of Canadian Grasslands Volumes 3 and 4: Biodiversity and Systematics Parts 1 and 2" by H.A. Cárcamo, and D.J. Giberson, 2014, 293

Gillott C. "A Buzz in the Meadow" by Dave Goulson, 2014, 296

John, R. "Field Guide to the Birds of Australia - A Photographic Guide" by Iain Campbell, Sam Woods, and Nick Leseberg, 2014, 98-99

John, R. "Birds of New Guinea: Second Edition" by Thane K. Pratt and Bruce M. Beehler, 2015, 99

John, R. "Birds and Animals of Australia's Top End: Darwin, Kakadu, Katherine, and Kununurra" by Nick Leseberg and Iain Campbell, 2015, 294

John, R. "Field Guide to the Birds of Australia - A Photographic Guide" by Iain Campbell, Sam Woods, and Nick Leseberg, 2014, 294-295

Lauff, R. "Marmot Biology - Sociality, Individual Fitness, and Population Dynamics" by Kenneth B. Armitage, 2014, 104

John, R. "A Field Guide to Common Fishes of the Pacific Northwest" by Andy Lamb, Bernard Hanby, and Phil Edgell, 2015, 409

Lim, B.K. "An Indomitable Beast: The Remarkable Journey of the Jaguar" by Alan Rabinowitz, 2014, 104-105

O’Neill, J. "Marine Community Ecology and Conservation" by M.D. Bertness et al., 2014, 103-104

Smith, C.M. "The Sea Among Us: the Amazing Strait of Georgia" by Richard Beamish and Gordon McFarlane, 2014, 102-103

Way, J. "The Carnivore Way: Coexisting with and Conserving North America's Predators" by Cristina Eisenberg, 2014, 100-101

Way, J. "Wolves on the Hunt: The Behavior of Wolves Hunting Wild Prey" by L. David Mech, Douglas W. Smith, and Daniel R. MacNulty, 2015, 297-299 